\title{
A THEOREM OF PALEY-WIENER TYPE FOR SCHRÖDINGER EVOLUTIONS
}

\author{
C. E. KENIG, G. PONCE, AND L. VEGA
}

\begin{abstract}
We prove unique continuation principles for solutions of evolution Schrödinger equations with time dependent potentials. These correspond to uncertainly principles of Paley-Wiener type for the Fourier transform. Our results extends to a large class of semi-linear Schrödinger equation.
\end{abstract}

\section{INTRODUCTION}

In this paper we study unique continuation properties of solutions of Schrödinger equations of the form

$$
\partial_{t} u=i(\triangle u+V(x, t) u), \quad(x, t) \in \mathbb{R}^{n} \times[0, T], \quad T>0 .
$$

The goal is to obtain sufficient conditions on the behavior of the solution $u$ at two different times and on the potential $V$ which guarantee that $u \equiv 0$ in $\mathbb{R}^{n} \times[0, T]$. Under appropriate assumptions this result will extend to the difference $v=u_{1}-u_{2}$ of two solutions $u_{1}, u_{2}$ of semi-linear Schrödinger equation

$$
\partial_{t} u=i(\triangle u+F(u, \bar{u})),
$$

from which one can conclude that $u_{1} \equiv u_{2}$.

Defining the Fourier transform of a function $f$ as

$$
\widehat{f}(\xi)=(2 \pi)^{-n / 2} \int_{\mathbb{R}^{n}} e^{-i \xi \cdot x} f(x) d x .
$$

one has

$$
\begin{aligned}
u(x, t) & =e^{i t \Delta} u_{0}(x)=\int_{\mathbb{R}^{n}} \frac{e^{i|x-y|^{2} / 4 t}}{(4 \pi i t)^{n / 2}} u_{0}(y) d y \\
& =\frac{e^{i|x|^{2} / 4 t}}{(4 \pi i t)^{n / 2}} \int_{\mathbb{R}^{n}} e^{-2 i x \cdot y / 4 t} e^{i|y|^{2} / 4 t} u_{0}(y) d y \\
& =\frac{e^{i|x|^{2} / 4 t}}{(2 i t)^{n / 2}}\left(e^{i|\cdot|^{2} / 4 t} u_{0}\right)\left(\frac{x}{2 t}\right),
\end{aligned}
$$

where $e^{i t \Delta} u_{0}(x)$ denotes the free solution of the Schrödinger equation with data $u_{0}$

$$
\partial_{t} u=i \triangle u, \quad u(x, 0)=u_{0}(x), \quad(x, t) \in \mathbb{R}^{n} \times \mathbb{R} .
$$

1991 Mathematics Subject Classification. Primary: 35Q55.

Key words and phrases. Schrödinger evolutions, unique continuation.

The first and second authors are supported by NSF grants DMS-0968472 and DMS-1101499 respectively. The third author is supported by MEC grant MTM2004-03029. 
The identity (1.3) tells us that this kind of results for the free solution of the Schrödinger equation are closely related to uncertainty principles for the Fourier transform. In this regard, one has the well known result of G. H. Hardy [11:

$$
\begin{aligned}
& \text { If } f(x)=O\left(e^{-x^{2} / \beta^{2}}\right), \widehat{f}(\xi)=O\left(e^{-4 \xi^{2} / \alpha^{2}}\right) \text { and } \alpha \beta<4, \text { then } f \equiv 0, \\
& \text { and if } \alpha \beta=4 \text {, then } f(x)=c e^{-x^{2} / \beta^{2}} .
\end{aligned}
$$

Its extension to higher dimensions $n \geq 2$ was obtained in 21]. The following generalization in terms of the $L^{2}$-norm was established in [5]:

$$
\text { If } e^{\frac{|x|^{2}}{\beta^{2}}} f(x), e^{\frac{4|\xi|^{2}}{\alpha^{2}}} \widehat{f}(\xi) \in L^{2}\left(\mathbb{R}^{n}\right) \text {, and } \alpha \beta \leq 4 \text {, then } f \equiv 0 \text {. }
$$

In terms of the free solution of the Schrödinger equation the $L^{2}$-version of Hardy Uncertainty Principle says :

$$
\text { If } e^{\frac{|x|^{2}}{\beta^{2}}} u_{0}(x), e^{\frac{|x|^{2}}{\alpha^{2}}} e^{i t \Delta} u_{0}(x) \in L^{2}\left(\mathbb{R}^{n}\right), \text { and } \alpha \beta \leq 4 t \text {, then } u_{0} \equiv 0 .
$$

In 8 , the following result was proven:

Theorem. ([8]) Given any solution $u \in C\left([0, T]: L^{2}\left(\mathbb{R}^{n}\right)\right)$ of

$$
\partial_{t} u=i(\triangle u+V(x, t) u), \quad(x, t) \in \mathbb{R}^{n} \times[0, T],
$$

with $V \in L^{\infty}\left(\mathbb{R}^{n} \times[0, T]\right)$,

$$
\lim _{\rho \rightarrow+\infty}\|V\|_{L^{1}\left([0, T]: L^{\infty}\left(\mathbb{R}^{n} \backslash B_{\rho}\right)\right)}=0 .
$$

and

$$
e^{\frac{|x|^{2}}{\beta^{2}}} u_{0}, \quad e^{\frac{|x|^{2}}{\alpha^{2}}} e^{i T \Delta} u_{0} \in L^{2}\left(\mathbb{R}^{n}\right)
$$

with $\alpha \beta<4 T$, then $u_{0} \equiv 0$.

Notice that the above Theorem recovers the $L^{2}$-version of the Hardy Uncertainty Principle (1.4) for solutions of the IVP (1.5), except for the limiting case $\alpha \beta=$ $4 T$ for which the corresponding result was proven to fail, see [8. Also one has the uncertainty principle of the type first studied by G. W. Morgan in [18. The following result was proven in 12 for the one dimensional case $n=1$ and extended to higher dimension in [3] and [19]:

If $f \in L^{1}\left(\mathbb{R}^{n}\right) \cap L^{2}\left(\mathbb{R}^{n}\right)$ and $\int_{\mathbb{R}^{n}} \int_{\mathbb{R}^{n}}|f(x)||\widehat{f}(\xi)| e^{|x \cdot \xi|} d x d \xi<\infty$, then $f \equiv 0$.

In particular, using Young's inequality this implies :

If $f \in L^{1}\left(\mathbb{R}^{n}\right) \cap L^{2}\left(\mathbb{R}^{n}\right), p \in(1,2), 1 / p+1 / q=1, \alpha, \beta>0$, and

$$
\int_{\mathbb{R}^{n}}|f(x)| e^{\frac{\alpha^{p}|x|^{p}}{p}} d x+\int_{\mathbb{R}^{n}}|\widehat{f}(\xi)| e^{\frac{\beta^{q}|\xi| q^{q}}{q}} d \xi<\infty, \quad \alpha \beta \geq 1 \Rightarrow f \equiv 0,
$$

or in terms of the solution of the free Schrödinger equation :

If $u_{0} \in L^{1}\left(\mathbb{R}^{n}\right) \cap L^{2}\left(\mathbb{R}^{n}\right)$ and for some $t \neq 0$

$$
\int_{\mathbb{R}^{n}}\left|u_{0}(x)\right| e^{\frac{\alpha^{p} \mid x x^{p}}{p}} d x+\int_{\mathbb{R}^{n}}\left|e^{i t \Delta} u_{0}(x)\right| e^{\frac{\beta^{q}|x|^{q}}{q(2 t)^{q}}} d x<\infty, \quad \alpha \beta \geq 1,
$$

then $u_{0} \equiv 0$.

In the one dimensional case $n=1$ the above condition $\alpha \beta \geq 1$ can be replaced by an optimal one $\alpha \beta>|\cos (p \pi / 2)|^{1 / p}$ as was established in [18, [1], [3]. 
In [9] the following result was obtained :

Theorem. (9]) Given $p \in(1,2)$ there exists $N_{p}>0$ such that if $u \in C\left([0,1]: L^{2}\left(\mathbb{R}^{n}\right)\right)$ is a solution of

$$
\partial_{t} u=i(\Delta u+V(x, t) u), \quad(x, t) \in \mathbb{R}^{n} \times[0,1],
$$

such that $V \in L^{\infty}\left(\mathbb{R}^{n} \times[0,1]\right)$,

$$
\lim _{\rho \rightarrow+\infty}\|V\|_{L^{1}\left([0,1]: L^{\infty}\left(\mathbb{R}^{n} \backslash B_{\rho}\right)\right)}=0,
$$

and there exist $\alpha, \beta>0$

$$
\int_{\mathbb{R}^{n}}|u(x, 0)|^{2} e^{2 \alpha^{p}|x|^{p} / p} d x+\int_{\mathbb{R}^{n}}|u(x, 1)|^{2} e^{2 \beta^{q}|x|^{q} / q} d x<\infty,
$$

with $1 / p+1 / q=1$ and

$$
\alpha \beta \geq N_{p}
$$

then $u \equiv 0$.

Some previous results concerning uniqueness properties of solutions of the Schrödinger equation were not directly motivated by the formula (1.3).

For solutions $u=u(x, t)$ of the 1-D cubic Schrödinger equation

$$
\partial_{t} u=i\left(\partial_{x}^{2} u \pm|u|^{2} u\right)
$$

B. Y. Zhang [23] showed :

If $u(x, t)=0$ for $(x, t) \in(-\infty, a) \times\{0,1\}($ or $(x, t) \in(a, \infty) \times\{0,1\})$ for some $a \in \mathbb{R}$, then $u \equiv 0$.

The proof is based on the inverse scattering method, which uses the fact that the equation in (1.11) is a completely integrable model.

In [17, under general assumptions on $F$ in (1.2), it was proven that :

If $u_{1}, u_{2} \in C\left([0,1]: H^{s}\left(\mathbb{R}^{n}\right)\right)$, with $s>\max \{n / 2 ; 2\}$ are solutions of the equation (1.2) with $F$ as in (1.2) such that

$$
u_{1}(x, t)=u_{2}(x, t), \quad(x, t) \in \Gamma_{x_{0}}^{c} \times\{0,1\},
$$

where $\Gamma_{x_{0}}^{c}$ denotes the complement of a cone $\Gamma_{x_{0}}$ with vertex $x_{0} \in \mathbb{R}^{n}$ and opening $<180^{\circ}$, then $u_{1} \equiv u_{2}$.

For further results in this direction see [16], 17], [14, [15, and references therein. Note that in 10 a unified approach was given to both kinds of results, using Lemma 3 and Corollary 1 below.

Returning to the uncertainty principle for the Fourier transform one has :

If $f \in L^{1}\left(\mathbb{R}^{n}\right)$ is non-zero and has compact support, then $\widehat{f}$ cannot satisfy a condition of the type $\widehat{f}(y)=O\left(e^{-\epsilon|y|}\right)$ for any $\epsilon>0$.

This is due to the fact that $\widehat{f}(y)=O\left(e^{-\epsilon|y|}\right)$ implies that $f$ has an analytic extension to the strip $\left\{z \in \mathbb{C}^{n}:|\operatorname{Im}(z)|<\epsilon\right\}$. However, it may be possible to have $f \in L^{1}\left(\mathbb{R}^{n}\right)$ a non-zero function with compact support, such that $\widehat{f}(\xi)=$ $O\left(e^{-\epsilon(y)|y|}\right), \epsilon(y)$ being a positive function tending to zero as $|y| \rightarrow \infty$. In the one-dimensional case $(n=1)$ A. E. Ingham [13] proved the following : 
There exists $f \in L^{1}(\mathbb{R})$ non-zero, even, vanishing outside an interval such that $\widehat{f}(y)=O\left(e^{-\epsilon(y)|y|}\right)$ with $\epsilon(y)$ being a positive function tending to zero at infinity if and only if

$$
\int_{a}^{\infty} \frac{\epsilon(y)}{y} d y<\infty, \quad \text { for some } \quad a>0
$$

In this regard the Paley-Wiener Theorem [20] gives a characterization of a function or distribution with compact support in term of the analyticity properties of its Fourier transform.

Our main result in this work is the following:

Theorem 1. Let $u \in C\left([0,1]: L^{2}\left(\mathbb{R}^{n}\right)\right)$ be a strong solution of the equation

$$
\partial_{t} u=i(\Delta u+V(x, t) u), \quad(x, t) \in \mathbb{R}^{n} \times[0,1] .
$$

Assume that

$$
\begin{gathered}
\sup _{0 \leq t \leq 1} \int_{\mathbb{R}^{n}}|u(x, t)|^{2} d x \leq A_{1}, \\
\int_{\mathbb{R}^{n}} e^{2 a_{1}\left|x_{1}\right|}|u(x, 0)|^{2} d x \leq A_{2}, \quad \text { for some } a_{1}>0, \\
\operatorname{supp} u(\cdot, 1) \subset\left\{x \in \mathbb{R}^{n}: x_{1} \leq a_{2}\right\}, \quad \text { for some } a_{2}<\infty,
\end{gathered}
$$

with

$$
V \in L^{\infty}\left(\mathbb{R}^{n} \times[0,1]\right), \quad\|V\|_{L^{\infty}\left(\mathbb{R}^{n} \times[0,1]\right)}=M_{0},
$$

and

$$
\lim _{\rho \rightarrow+\infty}\|V\|_{L^{1}\left([0,1]: L^{\infty}\left(\mathbb{R}^{n} \backslash B_{\rho}\right)\right)}=0
$$

Then $u \equiv 0$.

Remarks: (a) Note that in order to prove Theorem 1, by translation in $x_{1}$, we can choose who $a_{2}$ is. We will show that there exists $m>0$ (small) with the property that if (1.13), (1.14), (1.16), (1.17) hold and (1.15) holds with $a_{2}=m$, then

$$
u(x, 1)=0 \quad \text { for } \quad x \in \mathbb{R}^{n} \text { such that } m / 2<x_{1} \leq m .
$$

This clearly yields the desired result. Without loss of generality we will assume $m<1$.

(b) By rescaling it is clear that the result in Theorem 1 applies to any time interval $[0, T]$.

(c) We recall that in Theorem 1 there are no hypotheses on the size of the potential $V$ in the given class or on its regularity.

(d) A weaker version of Theorem 1 was announced in [10].

As a direct consequence of Theorem 1 we get the following result regarding the uniqueness of solutions for non-linear equations of the form (1.2).

Theorem 2. Given

$$
u_{1}, u_{2} \in C\left([0, T]: H^{k}\left(\mathbb{R}^{n}\right)\right), \quad 0<T \leq \infty,
$$


strong solutions of (1.2) with $k \in \mathbb{Z}^{+}, k>n / 2, F: \mathbb{C}^{2} \rightarrow \mathbb{C}, F \in C^{k}$ and $F(0)=\partial_{u} F(0)=\partial_{\bar{u}} F(0)=0$ such that

$$
\operatorname{supp}\left(u_{1}(\cdot, 0)-u_{2}(\cdot, 0)\right) \subset\left\{x \in \mathbb{R}^{n}: x_{1} \leq a_{2}\right\}, \quad a_{2}<\infty .
$$

If for some $t \in(0, T)$ and for some $\epsilon>0$

$$
u_{1}(\cdot, t)-u_{2}(\cdot, t) \in L^{2}\left(e^{\epsilon\left|x_{1}\right|} d x\right),
$$

then $u_{1} \equiv u_{2}$.

Remarks: (a) In particular, by taking $u_{2} \equiv 0$, Theorem 2 shows that if $u_{1}(\cdot, 0)$ has compact support, then for any $t \in(0, T) u_{1}(\cdot, t)$ cannot decay exponentially.

(b) In the case $F(u, \bar{u})=|u|^{\alpha-1} u$, with $\alpha>n / 2$ if $\alpha$ is not an odd integer, we have that if $\varphi$ is the unique non-negative, radially symmetric solution of

$$
-\Delta \varphi+\omega \varphi=|\varphi|^{\alpha-1} \varphi, \quad \omega>0,
$$

then

$$
u_{1}(x, t)=e^{i \omega t} \varphi(x)
$$

is a solution ("standing wave") of

$$
\partial_{t} u=i\left(\Delta u+|u|^{\alpha-1} u\right) .
$$

It was established in [22, 2] that there exist constants $c_{0}, c_{1}>0$ such that

$$
\varphi(x) \leq c_{0} e^{-c_{1}|x|} .
$$

Therefore, if we denote by $u_{2}(x, t)$ the solution of the equation (1.21) with $\alpha>n / 2$ and data $u_{2}(x, 0)=\varphi(x)+\phi(x), \phi \in H^{s}\left(\mathbb{R}^{n}\right), s>n / 2$ having compact support it follows from Theorem 2, (1.20) and (1.22) that for any $t \neq 0$

$$
u_{2}(\cdot, t) \notin L^{2}\left(e^{\epsilon|x|} d x\right), \quad \text { for any } \quad \epsilon>0 .
$$

In general, the same result (1.23) applies (in the time interval $[0, T]$ ) if one assumes that $u_{1}$ is a solution of (1.20) having exponential decay

$$
\left|u_{1}(x, t)\right| \leq c_{0} e^{-c_{1}|x|}, \quad c_{0}, c_{1}>0 \quad(x, t) \in \mathbb{R}^{n} \times[0, T],
$$

and $u_{2}$ is the solution of (1.20) corresponding to an initial data

$$
u_{2}(x, 0)=u_{1}(x, 0)+\phi(x), \quad \phi \in H^{s}\left(\mathbb{R}^{n}\right), \quad s>n / 2 \quad \text { with compact support. }
$$

The rest of this paper is organized as follows: section 2 contains all the preliminary results to be used in the proof of Theorem 1. A version of them has been proved in 9, 8, 10. However, in some cases modifications are needed to apply them in the setting considered here. Hence, some of their proofs will be sketched. Section 3 contains the proof of Theorem 1

\section{Preliminary Estimates}

In this section we describe the estimates to be used in the proof of Theorem 1.

First we recall a key step in the uniform exponential decay estimate established in [17: 
Lemma 1. There exists $\epsilon_{n}>0$ such that if

$$
\mathbb{V}: \mathbb{R}^{n} \times[0,1] \rightarrow \mathbb{C}, \quad \text { with } \quad\|\mathbb{V}\|_{L_{t}^{1} L_{x}^{\infty}} \leq \epsilon_{n},
$$

and $u \in C\left([0,1]: L^{2}\left(\mathbb{R}^{n}\right)\right)$ is a strong solution of the IVP

$$
\left\{\begin{array}{l}
\partial_{t} u=i(\Delta+\mathbb{V}(x, t)) u+\mathbb{G}(x, t), \\
u(x, 0)=u_{0}(x)
\end{array}\right.
$$

with

$$
u_{0}, u_{1} \equiv u(\cdot, 1) \in L^{2}\left(e^{2 \lambda \cdot x} d x\right), \quad \mathbb{G} \in L^{1}\left([0,1]: L^{2}\left(e^{2 \lambda \cdot x} d x\right)\right),
$$

for some $\lambda \in \mathbb{R}^{n}$, then there exists $c_{n}$ independent of $\lambda$ such that

$$
\begin{aligned}
& \sup _{0 \leq t \leq 1}\left\|e^{\lambda \cdot x} u(\cdot, t)\right\|_{L^{2}\left(\mathbb{R}^{n}\right)} \\
& \leq c_{n}\left(\left\|e^{\lambda \cdot x} u_{0}\right\|_{L^{2}\left(\mathbb{R}^{n}\right)}+\left\|e^{\lambda \cdot x} u_{1}\right\|_{L^{2}\left(\mathbb{R}^{n}\right)}+\int_{0}^{1}\left\|e^{\lambda \cdot x} \mathbb{G}(\cdot, t)\right\|_{L^{2}\left(\mathbb{R}^{n}\right)} d t\right) .
\end{aligned}
$$

Notice that in Lemma 1 one assumes the existence of a reference $L^{2}$ - solution $u$ of the equation (2.2) and gets a control on the decay of the solution in the whole time interval in terms of that at the end points and that of the "external force". In general, under appropriate assumptions on the potential $V(x, t)$ in (1.1) one writes

$$
V(x, t) u=\chi_{\rho} V(x, t) u+\left(1-\chi_{\rho}\right) V(x, t) u=\mathbb{V}(x, t) u+\mathbb{G}(x, t),
$$

with $\chi_{\rho} \in C_{0}^{\infty}, \chi_{\rho}(x)=1,|x|<\rho$, supported in $|x|<2 \rho$, and obtains the estimate (2.4) by fixing $\rho$ sufficiently large. Also under appropriate hypotheses on $F$ and $u$ a similar argument can be used for the semi-linear equation in (1.2).

Next, we recall the conformal or Appell transformation:

Lemma 2. If $u(y, s)$ verifies

$$
\partial_{s} u=i(\triangle u+V(y, s) u+F(y, s)), \quad(y, s) \in \mathbb{R}^{n} \times[0,1],
$$

and $\alpha$ and $\beta$ are positive, then

$$
\widetilde{u}(x, t)=\left(\frac{\sqrt{\alpha \beta}}{\alpha(1-t)+\beta t}\right)^{\frac{n}{2}} u\left(\frac{\sqrt{\alpha \beta} x}{\alpha(1-t)+\beta t}, \frac{\beta t}{\alpha(1-t)+\beta t}\right) e^{\frac{(\alpha-\beta)|x|^{2}}{4 i(\alpha(1-t)+\beta t)}},
$$

verifies

$$
\partial_{t} \widetilde{u}=i(\triangle \widetilde{u}+\widetilde{V}(x, t) \widetilde{u}+\widetilde{F}(x, t)), \quad(x, t) \in \mathbb{R}^{n} \times[0,1],
$$

with

$$
\widetilde{V}(x, t)=\frac{\alpha \beta}{(\alpha(1-t)+\beta t)^{2}} V\left(\frac{\sqrt{\alpha \beta} x}{\alpha(1-t)+\beta t}, \frac{\beta t}{\alpha(1-t)+\beta t}\right),
$$

and

$$
\widetilde{F}(x, t)=\left(\frac{\sqrt{\alpha \beta}}{\alpha(1-t)+\beta t}\right)^{\frac{n}{2}+2} F\left(\frac{\sqrt{\alpha \beta} x}{\alpha(1-t)+\beta t}, \frac{\beta t}{\alpha(1-t)+\beta t}\right) e^{\frac{(\alpha-\beta)|x|^{2}}{4 i(\alpha(1-t)+\beta t)}} .
$$

The following result is a modified version of the one in [6] (Lemma 3.1, page 1818). It will provide a needed lower bound of the $L^{2}$-norm of the solution of the equation (1.1) and its first order derivatives in the $x_{1}$-variable in the domain $\left\{x: R-1<x_{1}<R\right\} \times[0,1]$. 
Lemma 3. Assume that $R>0$ large enough and that $\varphi:[0,1] \rightarrow \mathbb{R}$ is a smooth function. Then, there exists $c=c\left(n ;\left\|\varphi^{\prime}\right\|_{\infty}+\left\|\varphi^{\prime \prime}\right\|_{\infty}\right)>0$ such that the inequality

$$
\frac{\sigma^{3 / 2}}{R^{2}}\left\|e^{\sigma\left|\frac{x_{1}-x_{0,1}}{R}+\varphi(t)\right|^{2}} g\right\|_{L^{2}(d x d t)} \leq c\left\|e^{\sigma\left|\frac{x_{1}-x_{0,1}}{R}+\varphi(t)\right|^{2}}\left(i \partial_{t}+\Delta\right) g\right\|_{L^{2}(d x d t)}
$$

holds when $\sigma \geq c R^{2}$ and $g \in C_{0}^{\infty}\left(\mathbb{R}^{n+1}\right)$ is supported on the set

$$
\left\{(x, t)=\left(x_{1}, . ., x_{n}, t\right) \in \mathbb{R}^{n+1}:\left|\frac{x_{1}-x_{0,1}}{R}+\varphi(t)\right| \geq 1\right\} .
$$

Proof. As it was remarked above this result is a variation of the one given in detail in [6], hence a sketch will suffice.

By translation, without loss of generality, we can assume $x_{0,1}=0$. Let

$$
f(x, t)=e^{\sigma\left|\frac{x_{1}}{R}+\varphi(t)\right|^{2}} g(x, t) .
$$

Then,

$$
e^{\sigma\left|\frac{x_{1}}{R}+\varphi(t)\right|^{2}}\left(i \partial_{t}+\Delta\right) g=S_{\sigma} f-4 \sigma A_{\sigma} f
$$

where

$$
\begin{aligned}
& S_{\sigma}=i \partial_{t}+\Delta+\frac{4 \sigma^{2}}{R^{2}}\left|\frac{x_{1}}{R}+\varphi\right|^{2} \\
& A_{\sigma}=\frac{1}{R}\left(\frac{x_{1}}{R}+\varphi\right) \partial_{x_{1}}+\frac{1}{2 R^{2}}+\frac{i \varphi^{\prime}}{2}\left(\frac{x_{1}}{R}+\varphi\right) .
\end{aligned}
$$

Thus,

$$
S_{\sigma}^{*}=S_{\sigma}, \quad A_{\sigma}^{*}=-A_{\sigma},
$$

and integrating by parts (possible since $g \in C_{0}^{\infty}\left(\mathbb{R}^{n+1}\right)$ ) one sees that

$$
\begin{aligned}
\left\|e^{\sigma\left|\frac{x_{1}}{R}+\varphi\right|^{2}}\left(i \partial_{t}+\Delta\right) g\right\|_{2}^{2}=\left\langle S_{\sigma} f-4 \sigma A_{\sigma} f, S_{\sigma} f-4 \sigma A_{\sigma} f\right\rangle \\
\geq-4 \sigma\left\langle\left(S_{\sigma} A_{\sigma}-A_{\sigma} S_{\sigma}\right) f, f\right\rangle=-4 \sigma\left\langle\left[S_{\sigma}, A_{\sigma}\right] f, f\right\rangle .
\end{aligned}
$$

A calculation shows that

$$
\left[S_{\sigma}, A_{\sigma}\right]=\frac{2}{R^{2}} \partial_{x_{1}}^{2}-\frac{4 \sigma^{2}}{R^{4}}\left|\frac{x_{1}}{R}+\varphi\right|^{2}-\frac{1}{2}\left[\left(\frac{x_{1}}{R}+\varphi\right) \varphi^{\prime \prime}+\left(\varphi^{\prime}\right)^{2}\right]+\frac{2 i \varphi^{\prime}}{R} \partial_{x_{1}} .
$$

From this it follows that

$$
\begin{aligned}
& \left\|e^{\sigma\left|\frac{x_{1}}{R}+\varphi\right|^{2}}\left(i \partial_{t}+\Delta\right) g\right\|_{2}^{2} \\
& \quad \geq \frac{16 \sigma^{3}}{R^{4}} \int\left|\frac{x_{1}}{R}+\varphi\right|^{2}|f|^{2} d x d t+\frac{8 \sigma}{R^{2}} \int\left|\partial_{x_{1}} f\right|^{2} d x d t \\
& \quad+2 \sigma \int\left[\left(\frac{x_{1}}{R}+\varphi\right) \varphi^{\prime \prime}+\left(\varphi^{\prime}\right)^{2}\right]|f|^{2} d x d t-\Im\left(\frac{8 \sigma i}{R} \int \varphi^{\prime} \partial_{x_{1}} f \bar{f} d x d t\right) .
\end{aligned}
$$

Now, when $\sigma \geq c R^{2}$ one has

$$
\frac{\sigma^{3}}{R^{4}} \geq c^{2} \sigma,
$$

so by taking $c$ large enough, depending on $\left\|\varphi^{\prime}\right\|_{\infty}$ and $\left\|\varphi^{\prime \prime}\right\|_{\infty}$, and using that $\left|\frac{x_{1}}{R}+\varphi(t)\right| \geq 1$ on the $\operatorname{supp}(f)=\operatorname{supp}(g)$, we can hide the third term on the right hand side (r.h.s.) in the inequality (2.13) in the first term in the r.h.s. Also, since

$$
\begin{aligned}
& \left|\frac{8 \sigma i}{R} \int\right| \varphi^{\prime}|| \partial_{x_{1}} f|| \bar{f}|d x d t| \\
& \leq \frac{8 \sigma}{R}\left\|\varphi^{\prime}\right\|_{\infty} \int|f|\left|\partial_{x_{1}} f\right| \leq 4 \sigma\left\|\varphi^{\prime}\right\|_{\infty}^{2} \int|f|^{2} d x d t+\frac{4 \sigma}{R^{2}} \int\left|\partial_{x_{1}} f\right|^{2} d x d t
\end{aligned}
$$


the contribution of this term in (2.13) can be hidden by the first and second term in the r.h.s. of (2.13) if $c$ is large. This concludes the proof.

Note that the same proof works by taking $c$ a bit larger, if we only assume $\left|\frac{x_{1}}{R}+\varphi(t)\right| \geq 1 / 2$ on $\operatorname{supp}(g)$.

In the proof of Theorem 1 we shall need the following extension of Lemma 3

Corollary 1. Assume $g \in L^{2}\left(\mathbb{R}^{n+1}\right)$ with $x_{1}, t$ on $\operatorname{supp}(g)$ bounded,

$$
\operatorname{supp}(g) \subset\left\{(x, t)=\left(x_{1}, . ., x_{n}, t\right) \in \mathbb{R}^{n+1}:\left|\frac{x_{1}-x_{0,1}}{R}+\varphi(t)\right| \geq 1\right\}
$$

and $\left(i \partial_{t}+\Delta\right) g \in L^{2}\left(\mathbb{R}^{n+1}\right)$, then the inequality (2.10) holds.

Proof. We can again assume that $x_{0,1}=0$. We introduce the notation $x=\left(x_{1}, x^{\prime}\right) \in$ $\mathbb{R} \times \mathbb{R}^{n-1}$. Let $\eta_{1} \in C_{0}^{\infty}(\mathbb{R}), \eta_{1} \geq 0, \operatorname{supp}\left(\eta_{1}\right) \subset\left\{\left|x_{1}\right|<1\right\}$ and $\eta_{2} \in C_{0}^{\infty}\left(\mathbb{R}^{n-1}\right)$, $\eta_{2} \geq 0, \operatorname{supp}\left(\eta_{2}\right) \subset\left\{\left|x^{\prime}\right|<1\right\}$ with

$$
\int_{\mathbb{R}} \eta_{1}\left(x_{1}\right) d x_{1}=1 \quad \text { and } \quad \int_{\mathbb{R}^{n-1}} \eta_{2}\left(x^{\prime}\right) d x^{\prime}=1 .
$$

For $\delta>0$ small define

$$
h_{\delta}(x, t)=\frac{1}{\delta^{n+2}} \eta_{1}\left(t / \delta^{2}\right) \eta_{1}\left(x_{1} / \delta\right) \eta_{2}\left(x^{\prime} / \delta^{n-1}\right) \quad \text { and } \quad g_{\delta}=h_{\delta} * g .
$$

Let $\theta \in C_{0}^{\infty}\left(\mathbb{R}^{n-1}\right), \theta\left(x^{\prime}\right)=1,\left|x^{\prime}\right| \leq 1$, and $\operatorname{supp}(\theta) \subset\left\{\left|x^{\prime}\right|<2\right\}$. For $l$ large, define

$$
g_{\delta, l}(x, t)=\theta\left(x^{\prime} / l\right) g_{\delta}(x, t) .
$$

Note that for $\delta>0$ small,

$$
\operatorname{supp}\left(g_{\delta}\right) \subset\left\{(x, t):\left|\frac{x_{1}}{R}+\varphi(t)\right|^{2} \geq 1 / 2\right\},
$$

and the same holds for $g_{\delta, l}$. Moreover, $g_{\delta, l} \in C_{0}^{\infty}\left(\mathbb{R}^{n+1}\right)$.

We apply Lemma 3 to $g_{\delta, l}$ to obtain:

$$
\frac{\sigma^{3 / 2}}{R^{2}}\left\|e^{\sigma\left|\frac{x_{1}}{R}+\varphi(t)\right|^{2}} g_{\delta, l}\right\|_{L^{2}(d x d t)} \leq c\left\|e^{\sigma\left|\frac{x_{1}}{R}+\varphi(t)\right|^{2}}\left(i \partial_{t}+\Delta\right) g_{\delta, l}\right\|_{L^{2}(d x d t)} .
$$

Next, we fix $\delta>0$ small and see that

$$
\begin{aligned}
\left(i \partial_{t}+\Delta\right) g_{\delta, l} & =\theta\left(x^{\prime} / l\right)\left(i \partial_{t}+\Delta\right) g_{\delta} \\
& +\frac{2}{l} \nabla^{\prime} \theta\left(x^{\prime} / l\right) \cdot \nabla^{\prime} g_{\delta}(x, t)+\frac{1}{l^{2}} \Delta \theta\left(x^{\prime} / l\right) g_{\delta}(x, t) .
\end{aligned}
$$

Therefore, by taking $l \rightarrow \infty$ the $L^{2}(d x d t)$-norm of the the last two terms on the r.h.s. of (2.15) tend to zero. Hence, inserting this in (2.14) we obtain the same estimate for $g_{\delta}$. Next, we have that

$$
\left(i \partial_{t}+\Delta\right) g_{\delta}=\left(i \partial_{t}+\Delta\right)\left(h_{\delta} * g\right)=h_{\delta} *\left(i \partial_{t}+\Delta\right) g .
$$

Using the supremum in $\delta$ (non-isotropic maximal function) and its boundedness, together with the boundedness of the support in $\left(x_{1}, t\right)$ of $g$, so that

$$
e^{\sigma\left|x_{1} / R+\varphi(t)\right|^{2}} \leq c_{\sigma, R},
$$

by the dominated convergence theorem we can pass to the limit as $\delta \rightarrow 0$ to obtain the desired result. 


\section{Proof of Theorem 1}

We divide our argument into six steps:

Step 1: We claim that

$$
\sup _{0 \leq t \leq 1} \int_{\mathbb{R}^{n}} e^{2 a_{1} x_{1}}|u(x, t)|^{2} d x \leq A_{3} .
$$

$\underline{\text { Proof of Step } 1}$ : Using (1.17) in Theorem 1 we choose $\rho$ so large such that

$$
\left\|V \chi_{\{|x| \geq \rho\}}\right\|_{L^{1}\left([0,1]: L^{\infty}\left(\mathbb{R}^{n}\right)\right)} \leq \epsilon_{n}
$$

with $\epsilon_{n}$ as in Lemma 1 . From (1.13)-(1.15) we have

$$
\int_{\mathbb{R}^{n}} e^{2 a_{1} x_{1}}|u(x, 0)|^{2} d x \leq A_{2},
$$

and

$$
\int_{\mathbb{R}^{n}} e^{2 a_{1} x_{1}}|u(x, 1)|^{2} d x \leq A_{1}+e^{2 a_{1} m} \leq A_{1}+e^{2 a_{1}} .
$$

We apply Lemma 1, with $\mathbb{G}(x, t)=-\chi_{\{|x| \leq \rho\}} V(x, t) u(x, t)$, using that

$$
\int_{0}^{1}\left\|e^{a_{1} x_{1}} \chi_{\{|x| \leq \rho\}} V u\right\|_{2} d t \leq e^{a_{1} \rho} M_{0} A_{1},
$$

which gives step 1 with $A_{3}=A_{3}\left(A_{1} ; A_{2} ; a_{1} ; M_{0} ; \rho\right)$.

Step 2: Define $\delta>0$ as

$$
\delta=\frac{\epsilon_{n}}{M_{0}+1}
$$

with $M_{0}$ as in (1.16) and $\epsilon_{n}$ as in Lemma 1. Note that $\delta<1$, and

$$
\int_{1-\delta}^{1}\|V(\cdot, t)\|_{\infty} d t \leq \epsilon_{n}
$$

Let

$$
v(x, t)=u\left(\delta^{1 / 2} x, \delta t+1-\delta\right) .
$$

We shall show that under the hypothesis of Theorem 1

$$
\int_{\frac{m}{2 \delta^{1 / 2}}<x_{1}<\frac{m}{\delta^{1 / 2}}}|v(x, 1)|^{2} d x=\int_{\frac{m}{2}<x_{1}<m}|u(x, 1)|^{2} d x=0
$$

as desired.

Defining

$$
V_{\delta}(x, t)=\delta V\left(\delta^{1 / 2} x, \delta t+1-\delta\right)
$$

we see that $v(x, t)$ satisfies the equation

$$
\partial_{t} v=i\left(\Delta v+V_{\delta} v\right), \quad(x, t) \in \mathbb{R}^{n} \times[0,1] .
$$

We notice, using (3.3), that

$$
\left\|V_{\delta}\right\|_{L^{\infty}\left(\mathbb{R}^{n} \times[0,1]\right)} \leq M_{0} \delta \leq \epsilon_{n}, \quad \int_{0}^{1}\left\|V_{\delta}(\cdot, t)\right\|_{\infty} d t \leq \epsilon_{n},
$$

and

$$
\int_{\mathbb{R}^{n}}|v(x, t)|^{2} d x=\frac{1}{\delta^{n / 2}} \int_{\mathbb{R}^{n}}|u(y, \delta t+1-\delta)|^{2} d y \leq \frac{A_{1}}{\delta^{n / 2}},
$$


with

Thus, from (3.1)

$$
\operatorname{supp}(v(\cdot, 1)) \subset\left\{x_{1} \leq m / \delta^{1 / 2}\right\} .
$$

$$
\int_{\mathbb{R}^{n}} e^{2 a_{1} x_{1} \delta^{1 / 2}}|v(x, 0)|^{2} d x=\int_{\mathbb{R}^{n}} e^{2 a_{1} x_{1} \delta^{1 / 2}}\left|u\left(\delta^{1 / 2} x, 1-\delta\right)\right|^{2} d x \leq \frac{A_{3}}{\delta^{n / 2}} .
$$

We remark that $\delta$ was fixed in (3.2) (independent of $m$ ), and that we can still choose $m$ small.

Step 3: Using the Appell (conformal) transformation Lemma 2 we have that if

$$
\partial_{s} v=i\left(\Delta v+V_{\delta} v\right), \quad(y, s) \in \mathbb{R}^{n} \times[0,1],
$$

then for any $\alpha, \beta>0$

$$
\widetilde{v}(x, t)=\left(\frac{\sqrt{\alpha \beta}}{\alpha(1-t)+\beta t}\right)^{\frac{n}{2}} v\left(\frac{\sqrt{\alpha \beta} x}{\alpha(1-t)+\beta t}, \frac{\beta t}{\alpha(1-t)+\beta t}\right) e^{\frac{(\alpha-\beta)|x|^{2}}{4 i(\alpha(1-t)+\beta t)}},
$$

verifies

$$
\partial_{t} \widetilde{v}=i(\Delta \widetilde{v}+\widetilde{V} \widetilde{v}), \quad(x, t) \in \mathbb{R}^{n} \times[0,1]
$$

with

$$
\widetilde{V}(x, t)=\frac{\alpha \beta}{(\alpha(1-t)+\beta t)^{2}} V_{\delta}\left(\frac{\sqrt{\alpha \beta} x}{\alpha(1-t)+\beta t}, \frac{\beta t}{\alpha(1-t)+\beta t}\right) .
$$

For $\lambda>0$ given we will choose $\alpha=\alpha(\lambda, \delta), \beta=\beta(\lambda, \delta)$. We recall that

$$
\left\|e^{a_{1} x_{1} \delta^{1 / 2}} v(\cdot, 0)\right\|_{2}^{2} \leq \frac{A_{3}}{\delta^{n / 2}},
$$

and from the support hypothesis

$$
\left\|e^{\lambda x_{1}} v(\cdot, 1)\right\|_{2}^{2} \leq \frac{e^{2 m \lambda / \delta^{1 / 2}} A_{1}}{\delta^{n / 2}} .
$$

We want $\gamma=\gamma(\lambda, \delta)$ such that

$$
\left\|e^{\gamma x_{1}} \widetilde{v}(x, 0)\right\|_{2}=\left\|e^{\gamma(\alpha / \beta)^{1 / 2} x_{1}} v(x, 0)\right\|_{2}=\left\|e^{a_{1} x_{1} \delta^{1 / 2}} v(\cdot, 0)\right\|_{2} \leq \frac{A_{3}^{1 / 2}}{\delta^{n / 4}},
$$

and

$$
\left\|e^{\gamma x_{1}} \widetilde{v}(x, 1)\right\|_{2}=\left\|e^{\gamma(\beta / \alpha)^{1 / 2} x_{1}} v(x, 1)\right\|_{2}=\left\|e^{\lambda x_{1}} v(\cdot, 1)\right\|_{2} \leq \frac{e^{\lambda m / \delta^{1 / 2}} A_{1}^{1 / 2}}{\delta^{n / 4}} .
$$

Thus, we choose

$$
\gamma(\alpha / \beta)^{1 / 2}=\delta^{1 / 2} a_{1}, \quad \gamma(\beta / \alpha)^{1 / 2}=\lambda,
$$

i.e.

$$
\gamma=\left(\lambda \delta^{1 / 2} a_{1}\right)^{1 / 2}, \quad \beta=\lambda, \quad \alpha=\delta^{1 / 2} a_{1} .
$$

Next, using the change of variable

$$
\widehat{t}=\frac{\beta}{\alpha(1-t)+\beta t}, \quad \widehat{d t}=\frac{\alpha \beta}{(\alpha(1-t)+\beta t)^{2}} d t,
$$


it follows that

$$
\begin{aligned}
& \int_{0}^{1}\|\tilde{V}(\cdot, t)\|_{\infty} d t \\
& =\int_{0}^{1}\left\|\frac{\alpha \beta}{(\alpha(1-t)+\beta t)^{2}} V_{\delta}\left(\frac{\sqrt{\alpha \beta} x}{\alpha(1-t)+\beta t}, \frac{\beta t}{\alpha(1-t)+\beta t}\right)\right\|_{\infty} d t \\
& =\int_{0}^{1}\left\|V_{\delta}(\cdot, \widehat{t})\right\|_{\infty} d \widehat{t} \leq \epsilon_{n},
\end{aligned}
$$

using (3.7). So we can apply Lemma 1 again, this time with $\mathbb{G} \equiv 0$, to obtain that

$$
\begin{aligned}
\sup _{0 \leq t \leq 1}\left\|e^{\gamma x_{1}} \widetilde{v}(\cdot, t)\right\|_{2} & \leq c_{n}\left(\frac{A_{3}^{1 / 2}}{\delta^{n / 4}}+\frac{A_{1}^{1 / 2}}{\delta^{n / 4}} e^{\lambda m / \delta^{1 / 2}}\right) \\
& \leq c_{\delta, a_{1}, A_{1}, A_{3}} e^{\lambda m / \delta^{1 / 2}} \\
& \leq c e^{\lambda m / \delta^{1 / 2}},
\end{aligned}
$$

if $\lambda>0$ is large and $A_{1} \neq 0$, (how large $\lambda$ is for this depends on $m, A_{1}, A_{3}$ and $\delta$, but this will not matter). Note that

$$
\|\widetilde{v}(\cdot, t)\|_{2}^{2}=\left\|v\left(\cdot, \frac{\beta t}{\alpha(1-t)+\beta t}\right)\right\|_{2}^{2} \leq \frac{A_{1}}{\delta^{n / 2}},
$$

hence

$$
\sup _{0 \leq t \leq 1}\|\widetilde{v}(\cdot, t)\|_{2} \leq \frac{A_{1}^{1 / 2}}{\delta^{n / 4}} .
$$

Now, we denote by $\phi_{0}\left(x_{1}\right) \geq 0$ a $C^{\infty}$ convex function such that

$$
\phi_{0}\left(x_{1}\right)= \begin{cases}0, & x_{1} \leq 0 \\ x_{1}-1 / 4, & x_{1} \geq 1 / 2\end{cases}
$$

and define

$$
\phi\left(x_{1}\right)=\left(1+\left(\phi\left(x_{1}\right)\right)^{2}\right)^{1 / 2} .
$$

Since $\gamma=\left(\lambda \delta^{1 / 2} a_{1}\right)^{1 / 2}$, from (3.11) and large $\lambda$ we have

$$
\sup _{0 \leq t \leq 1}\left\|e^{\gamma \phi\left(x_{1}\right)} \widetilde{v}(\cdot, t)\right\|_{2} \leq c_{\delta, a_{1}} e^{\lambda m / \delta^{1 / 2}} .
$$

A computation shows that

$$
\phi^{\prime}\left(x_{1}\right)=\frac{\phi_{0}\left(x_{1}\right) \phi_{0}^{\prime}\left(x_{1}\right)}{\left(1+\left(\phi_{0}\left(x_{1}\right)\right)^{2}\right)^{1 / 2}},
$$

and

$$
\phi^{\prime \prime}\left(x_{1}\right)=\frac{\left(\phi_{0}^{\prime}\left(x_{1}\right)\right)^{2}}{\left(1+\left(\phi_{0}\left(x_{1}\right)\right)^{2}\right)^{3 / 2}}+\frac{\phi_{0}\left(x_{1}\right) \phi_{0}^{\prime \prime}\left(x_{1}\right)}{\left(1+\left(\phi_{0}\left(x_{1}\right)\right)^{2}\right)^{3 / 2}} .
$$

Thus, for $x_{1} \geq 1 / 2$ one has that

$$
\phi^{\prime \prime}\left(x_{1}\right) \geq \frac{1}{4} \frac{1}{\left(1+x_{1}^{2}\right)^{3 / 2}}=\frac{1}{4} \frac{1}{\left\langle x_{1}\right\rangle^{3}} .
$$

We now follow an argument similar to that in [9] section 2. Let

$$
f(x, t)=e^{\gamma \phi\left(x_{1}\right)} \widetilde{v}(x, t) .
$$


Then $f$ verifies

$$
\partial_{t} f=\mathcal{S} f+\mathcal{A} f+i e^{\gamma \phi} F, \quad \text { in } \mathbb{R}^{n} \times[0,1],
$$

with symmetric and skew-symmetric operators $\mathcal{S}$ and $\mathcal{A}$

$$
\begin{aligned}
\mathcal{S} & =-i \gamma\left(2 \partial_{x_{1}} \phi \partial_{x_{1}}+\partial_{x_{1}}^{2} \phi\right), \\
\mathcal{A} & =i\left(\triangle+\gamma^{2}\left|\partial_{x_{1}} \phi\right|^{2}\right) .
\end{aligned}
$$

and

$$
F=\widetilde{V} \widetilde{v}
$$

A calculation shows that,

$$
\mathcal{S}_{t}+[\mathcal{S}, \mathcal{A}]=-\gamma\left[4 \partial_{x_{1}} \phi^{\prime \prime} \partial_{x_{1}}-4 \gamma^{2} \phi^{\prime \prime}\left(\phi^{\prime}\right)^{2}+\phi^{(4)}\right] .
$$

By Lemma 2 in 9

$$
\begin{aligned}
\partial_{t}^{2} H \equiv \partial_{t}^{2}(f, f)= & 2 \partial_{t} \operatorname{Re}\left(\partial_{t} f-\mathcal{S} f-\mathcal{A} f, f\right)+2\left(\mathcal{S}_{t} f+[\mathcal{S}, \mathcal{A}] f, f\right) \\
& +\left\|\partial_{t} f-\mathcal{A} f+\mathcal{S} f\right\|^{2}-\left\|\partial_{t} f-\mathcal{A} f-\mathcal{S} f\right\|^{2}
\end{aligned}
$$

so

$$
\begin{aligned}
\partial_{t}^{2} H & \geq 2 \partial_{t} \operatorname{Re}\left(\partial_{t} f-\mathcal{S} f-\mathcal{A} f, f\right) \\
& +2\left(\mathcal{S}_{t} f+[\mathcal{S}, \mathcal{A}] f, f\right)-\left\|\partial_{t} f-\mathcal{A} f-\mathcal{S} f\right\|^{2} .
\end{aligned}
$$

Multiplying (3.21) by $t(1-t)$ and integrating in $t$ we obtain

$$
\begin{aligned}
& 2 \int_{0}^{1} t(1-t)\left(\mathcal{S}_{t} f+[\mathcal{S}, \mathcal{A}] f, f\right) d t \\
& \leq c_{n} \sup _{[0,1]}\left\|e^{\gamma \phi} \widetilde{v}(t)\right\|_{2}^{2}+c_{n} \sup _{[0,1]}\left\|e^{\gamma \phi} F(t)\right\|_{2} .
\end{aligned}
$$

This computation can be justified by parabolic regularization using the fact that we already know the decay estimate for $\widetilde{v}$, see [7]. Note that for $\lambda$ sufficiently large

$$
\|\tilde{V}\|_{\infty} \leq\left(\frac{\beta}{\alpha}\right)\left\|V_{\delta}\right\|_{\infty} \leq \frac{\lambda}{\delta^{1 / 2} a_{1}} \delta M_{0}=\frac{\lambda \delta^{1 / 2} M_{0}}{a_{1}} .
$$

Hence, combining (3.11), (3.19), and (3.23) it follows that

$$
\begin{aligned}
& 8 \gamma \int_{0}^{1} \int t(1-t) \phi^{\prime \prime}\left(x_{1}\right)\left|\partial_{x_{1}} f\right|^{2} d x d t \\
& +8 \gamma^{3} \int_{0}^{1} \int t(1-t) \phi^{\prime \prime}\left(x_{1}\right)\left(\phi^{\prime}\left(x_{1}\right)\right)^{2}|f|^{2} d x d t \\
& \leq c_{n} \gamma \sup _{[0,1]}\|f(\cdot, t)\|_{2}^{2}+c_{\delta, M_{0}, a_{1}, n} \lambda \sup _{[0,1]}\|f(\cdot, t)\|_{2}^{2}+c_{n} \sup _{[0,1]}\|f(\cdot, t)\|_{2}^{2} \\
& \leq c_{\delta, M_{0}, a_{1}, n} \lambda e^{2 \lambda m / \delta^{1 / 2}} .
\end{aligned}
$$

We recall that

$$
\partial_{x_{1}} f=e^{\gamma \phi\left(x_{1}\right)} \partial_{x_{1}} \widetilde{v}+\gamma e^{\gamma \phi\left(x_{1}\right)} \phi^{\prime}\left(x_{1}\right) \widetilde{v},
$$


thus

$$
\begin{aligned}
& \gamma\left|\partial_{x_{1}} f\right|^{2}=\gamma e^{2 \gamma \phi\left(x_{1}\right)}\left|\partial_{x_{1}} \widetilde{v}+\gamma \phi^{\prime}\left(x_{1}\right) \widetilde{v}\right|^{2} \\
& =e^{2 \gamma \phi\left(x_{1}\right)}\left(\gamma\left|\partial_{x_{1}} \widetilde{v}\right|^{2}+2 \gamma^{2} \phi^{\prime}\left(x_{1}\right) \widetilde{v} \partial_{x_{1}} \widetilde{v}+\gamma^{3}\left(\phi^{\prime}\left(x_{1}\right)\right)^{2}|\widetilde{v}|^{2}\right),
\end{aligned}
$$

with

$$
\left|2 \gamma^{2} \phi^{\prime}\left(x_{1}\right) \widetilde{v} \partial_{x_{1}} \widetilde{v}\right| \leq \frac{1}{2} \gamma\left|\partial_{x_{1}} \widetilde{v}\right|^{2}+2 \gamma^{3}\left(\phi^{\prime}\left(x_{1}\right)\right)^{2}|\widetilde{v}|^{2} .
$$

Inserting these estimates in (3.24) for $\lambda$ large one gets

$$
4 \gamma \int_{0}^{1} \int t(1-t) \phi^{\prime \prime}\left(x_{1}\right) e^{2 \gamma \phi\left(x_{1}\right)}\left|\partial_{x_{1}} \widetilde{v}\right|^{2} d x d t \leq c_{\delta, M_{0}, a_{1}, n} \lambda e^{2 \lambda m / \delta^{1 / 2}} .
$$

Hence, for $x_{1}>1 / 2$ from (3.16) one has that

$$
\gamma \int_{0}^{1} \int t(1-t) \frac{1}{\left\langle x_{1}\right\rangle^{3}} e^{2 \gamma \phi\left(x_{1}\right)}\left|\partial_{x_{1}} \widetilde{v}\right|^{2} d x d t \leq c_{\delta, M_{0}, a_{1}, n} \lambda e^{2 \lambda m / \delta^{1 / 2}}
$$

for $\lambda$ large. Collecting the above information, (3.11), and (3.24) we conclude that

$$
\begin{aligned}
& \sup _{0 \leq t \leq 1}\left\|e^{\gamma \phi\left(x_{1}\right)} \widetilde{v}(\cdot, t)\right\|_{2}^{2}+\gamma \int_{0}^{1} \int_{x_{1}>\frac{1}{2}} t(1-t) \frac{1}{\left\langle x_{1}\right\rangle^{3}} e^{2 \gamma \phi\left(x_{1}\right)}\left|\partial_{x_{1}} \widetilde{v}\right|^{2} d x d t \\
& \leq c_{\delta, M_{0}, a_{1}, n} \lambda e^{2 \lambda m / \delta^{1 / 2}} .
\end{aligned}
$$

Step 4 : We will give lower bounds for

$$
\Phi=\int_{2 \leq x_{1} \leq R / 2} \int_{3 / 8}^{5 / 8}|\widetilde{v}(x, t)|^{2} d t d x
$$

for $R$ large to be chosen.

First, we recall that

$$
\Phi=\int_{2 \leq x_{1} \leq R / 2} \int_{3 / 8}^{5 / 8}\left|\left(\frac{\sqrt{\alpha \beta}}{\alpha(1-t)+\beta t}\right)^{\frac{n}{2}} v\left(\frac{\sqrt{\alpha \beta} x}{\alpha(1-t)+\beta t}, \frac{\beta t}{\alpha(1-t)+\beta t}\right)\right|^{2} d x d t .
$$

Next, for $t \in[3 / 8,5 / 8]$ we see that

$$
s(t)=\frac{\beta t}{\alpha(1-t)+\beta t},
$$

satisfies that

$$
d t=\frac{(\alpha(1-t)+\beta t)^{2}}{\alpha \beta} d s \simeq \frac{\beta^{2}}{\alpha \beta} d s=\frac{\beta}{\alpha} d s,
$$

with

$$
s(3 / 8)=\frac{3 \beta}{5 \alpha+3 \beta} \in(1 / 2,1),
$$

and

$$
s(5 / 8)=\frac{5 \beta}{3 \alpha+5 \beta} \in(1 / 2,1) .
$$

Therefore

$$
s(5 / 8)-s(3 / 8)=\frac{2 \alpha \beta}{(5 \alpha+3 \beta)(3 \alpha+5 \beta)} \cong \frac{\alpha}{\beta},
$$

for large $\lambda$, and

$$
s(5 / 8)>s(3 / 8) \uparrow 1 \quad \text { as } \quad \lambda \uparrow \infty .
$$


In the $x$-variable we have

$$
y=\frac{\sqrt{\alpha \beta}}{\alpha(1-t)+\beta} x,
$$

so for $t \in[3 / 8,5 / 8]$ and $2<x_{1}<R / 2$ one basically has that

$$
y_{1} \in\left[2 \sqrt{\frac{\alpha}{\beta}}, \frac{R}{2} \sqrt{\frac{\alpha}{\beta}}\right] \equiv A \text {. }
$$

Thus,

$$
\Phi \geq c_{n} \frac{\beta}{\alpha} \int_{A} \int_{I_{\lambda}}|v(y, s)|^{2} d s d y
$$

with

$$
I_{\lambda}=[s(3 / 8), s(5 / 8)], \quad\left|I_{\lambda}\right| \cong \frac{\alpha}{\beta} \quad \text { for } \quad \lambda>>1,
$$

with

$$
s(3 / 8) \rightarrow 1 \text { as } \lambda \uparrow \infty .
$$

We choose

$$
R=\frac{2 M \lambda^{1 / 2} m}{\left(\delta^{1 / 2} a_{1}\right)^{1 / 2} c_{n}}
$$

with

$$
\frac{2}{c_{n}} M \geq \frac{1}{\delta^{1 / 2}}
$$

to be fixed latter. Since

$$
2 \sqrt{\frac{\alpha}{\beta}}=2 \frac{\delta^{1 / 4} a_{1}^{1 / 2}}{\lambda^{1 / 2}} \rightarrow 0 \quad \text { as } \quad \lambda \uparrow \infty,
$$

and

$$
\frac{R}{2} \sqrt{\frac{\alpha}{\beta}}=\frac{2 M m}{c_{n}} \geq \frac{m}{c_{n} \delta^{1 / 2}} .
$$

Hence, from (3.26) we can conclude

$$
\liminf _{\lambda \uparrow \infty} \Phi \geq c_{n} \int_{0<y_{1}<\frac{m}{\delta^{1 / 2}}}|v(y, 1)|^{2} d y .
$$

$\underline{\text { Step } 5}$ : Upper bounds for

$$
\Xi(R) \equiv \int_{\frac{1}{2}<x_{1}<R} \int_{1 / 32}^{31 / 32}\left(|\widetilde{v}(x, t)|^{2}+\left|\partial_{x_{1}} \widetilde{v}(x, t)\right|^{2}\right) d t d x .
$$

For the square of the $L^{2}$-norm of $\widetilde{v}$ we have the bound $A_{1} / \delta^{n / 2}$, see (3.12). For the square of the $L^{2}$-norm of $\partial_{x_{1}} \widetilde{v}$ using the conclusion of Step 3 (3.25) we get the upper bound

$$
c_{\delta, M_{0}, a_{1}, n}\left(1+R^{3}\right) \lambda e^{2 \lambda m / \delta^{1 / 2}} .
$$

$\underline{\text { Step } 6}$ : Carleman estimate [4] and conclusion of the proof.

We assume that for $m>0$ to be chosen

$$
b \equiv \int_{\frac{m}{2 \delta^{1 / 2}}<y_{1}<\frac{m}{\delta^{1 / 2}}}|v(y, 1)|^{2} d y>0 .
$$


We recall that

$$
\operatorname{supp}(v(\cdot, 1)) \subset\left\{y_{1}<m / \delta^{1 / 2}\right\} .
$$

From step 4 we have that for $\lambda$ sufficiently large

$$
\int_{2 \leq x_{1} \leq R / 2} \int_{3 / 8}^{5 / 8}|\widetilde{v}(x, t)|^{2} d t d x \geq \frac{b}{2} .
$$

Now, let

$$
x_{0,1}=R / 2,
$$

and $\varphi:[0,1] \rightarrow \mathbb{R}$ be a smooth function such that $0 \leq \varphi(t) \leq 3 / 2-1 / R$,

$$
\varphi(t)=\left\{\begin{array}{lr}
3 / 2-1 / R, & t \in[3 / 8,5 / 8], \\
0, & t \in[0,1 / 4] \cup[3 / 4,1],
\end{array}\right.
$$

with $\varphi, \varphi^{\prime}, \varphi^{\prime \prime}$ uniformly bounded in $R$ for $R$ large. We fix

$$
\sigma=c R^{2},
$$

with $c$ denoting a universal constant whose value may change from line to line, so that Corollary 1 applies. Chose $\theta_{R} \in C^{\infty}(\mathbb{R})$, with $0 \leq \theta\left(x_{1}\right) \leq 1$ and

$$
\theta_{R}\left(x_{1}\right)= \begin{cases}1, & 1<x_{1}<R-1, \\ 0, & x_{1}<1 / 2 \text { or } x_{1}>R .\end{cases}
$$

Let $\zeta \in C^{\infty}(\mathbb{R})$ satisfy $0 \leq \zeta\left(x_{1}\right) \leq 1$ and

$$
\zeta\left(x_{1}\right)= \begin{cases}0, & x_{1}<1, \\ 1, & x_{1}>1+1 /(2 R) .\end{cases}
$$

Define

$$
g(x, t) \equiv \theta_{R}\left(x_{1}\right) \zeta\left(\frac{x_{1}-R / 2}{R}+\varphi(t)\right) \widetilde{v}(x, t) .
$$

Let us see that $g(x, t)$ verifies the hypotheses of Corollary 1 so we can apply the inequality (2.10). First, it is clear that it is supported on the set

$$
1 / 2<x_{1}<R, \quad 1 / 32<t<31 / 32, \quad\left|\frac{x_{1}-R / 2}{R}+\varphi(t)\right| \geq 1 .
$$

Below we shall see that

$$
\left(i \partial_{t}+\Delta\right) g \in L^{2}(d x d t)
$$

Note that

$$
\text { if } 3 / 2 \leq x_{1} \leq R-1 \quad \text { and } \quad 3 / 8 \leq t \leq 5 / 8 \text {, then } \quad g(x, t)=\widetilde{v}(x, t) .
$$

In this domain, $\theta_{R}\left(x_{1}\right) \equiv 1$, and

$$
\frac{x_{1}-R / 2}{R}+\varphi(t)=\frac{x_{1}}{R}+1-\frac{1}{R} \geq 1+\frac{1}{2 R},
$$

which gives (3.36).

Also if $x_{1}>2$ one has $x_{1} / R+1-1 / R \geq 1+1 / R$, so that we have a lower bound $\Gamma$ for the left hand side of (2.10) squared with

$$
\Gamma \equiv \frac{\sigma^{3}}{R^{4}} e^{2 \sigma(1+1 / R)^{2}} \int_{2<x_{1}<R-1} \int_{3 / 8}^{5 / 8}|\widetilde{v}(x, t)|^{2} d t d x \geq \frac{b}{2} c^{3} R^{2} e^{2 \sigma(1+1 / R)^{2}}
$$


for $R$ large from (3.31). The equation for $g$ is

$$
\begin{aligned}
& \left(i \partial_{t}+\Delta\right) g=\theta_{R}\left(x_{1}\right) \zeta\left(\frac{x_{1}-R / 2}{R}+\varphi(t)\right) \widetilde{V}(x, t) \widetilde{v} \\
& +\left[\zeta\left(\frac{x_{1}-R / 2}{R}+\varphi(t)\right)\left(2 \theta_{R}^{\prime}\left(x_{1}\right) \partial_{x_{1}} \widetilde{v}+\widetilde{v} \theta_{R}^{\prime \prime}\left(x_{1}\right)\right)\right] \\
& +\left[\left(i \zeta^{\prime}(\cdot) \varphi^{\prime}(t)+\zeta^{\prime \prime}(\cdot) \frac{1}{R^{2}}\right) \theta_{R}\left(x_{1}\right) \widetilde{v}+\frac{2}{R} \zeta^{\prime}(\cdot) \theta_{R}\left(x_{1}\right) \partial_{x_{1}} \widetilde{v}\right] \\
& \equiv E_{1}+E_{2}+E_{3} .
\end{aligned}
$$

Note that $\left(i \partial_{t}+\Delta\right) g \in L^{2}(d x d t)$ by Step 5 .

On the domain $1 / 2<x_{1}<R, 1 / 32<t<31 / 32$ (which contains the support of g) one has (see (3.10)

$$
\|\widetilde{V}\|_{\infty} \lesssim \frac{\alpha}{\beta} M_{0} \quad \text { for } \quad \lambda>>1
$$

Thus, since

$$
\frac{\sigma^{3}}{R^{4}}=c^{3} R^{2},
$$

for $R$ sufficiently large we can absorb the contribution of the term containing $E_{1}$ in the right hand side of (2.10) in the left hand side of (2.10). So we have

$$
\begin{aligned}
\frac{b}{2} c^{3} R^{2} e^{2 \sigma(1+1 / R)^{2}} & \leq c \iint\left|E_{2}\right|^{2} e^{2 \sigma\left|\frac{x_{1}-R / 2}{R}+\varphi(t)\right|^{2}} d x d t \\
& +c \iint\left|E_{3}\right|^{2} e^{2 \sigma\left|\frac{x_{1}-R / 2}{R}+\varphi(t)\right|^{2}} d x d t .
\end{aligned}
$$

Next, we analyze the contribution of $E_{2}$. In this case, each term contains a factor equal to a derivative of $\theta_{R}$, so the possible contribution are from the sets :

$1 / 2<x_{1}<1$ and $R-1<x_{1}<R$. If $1 / 2<x_{1}<1$, then

$$
\frac{x_{1}-R / 2}{R}+\varphi(t) \leq \frac{1}{R}-\frac{1}{2}+\frac{3}{2}-\frac{1}{R}=1,
$$

so in this domain

$$
\zeta\left(\frac{x_{1}-R / 2}{R}+\varphi(t)\right) \equiv 0
$$

In the region $R-1<x_{1}<R$ we have

$$
\frac{x_{1}-R / 2}{R}+\varphi(t) \leq 1-\frac{1}{2}+\frac{3}{2}-\frac{1}{R}=2-\frac{1}{R},
$$

so the contribution of the term involving $E_{2}$ is bounded above by

$$
\begin{aligned}
& \int_{1 / 32}^{31 / 32} \int_{R-1<x_{1}<R}\left(|\widetilde{v}|^{2}+\left|\partial_{x_{1}} \widetilde{v}\right|^{2}\right) e^{2 \sigma(1-1 / R)^{2}} d x d t \\
& =e^{2 \sigma(2-1 / R)^{2}} \int_{1 / 32}^{31 / 32} \int_{R-1<x_{1}<R}\left(|\widetilde{v}|^{2}+\left|\partial_{x_{1}} \widetilde{v}\right|^{2}\right) d x d t \\
& =\Xi(R) e^{2 \sigma(2-1 / R)^{2}} .
\end{aligned}
$$

Next, we consider the term involving $E_{3}$. In this case, each term contains a factor equal to a derivative of $\zeta$ so its support is restricted to

$$
1 \leq \frac{x_{1}-R / 2}{R}+\varphi(t) \leq 1+1 / 2 R,
$$


with $1 / 2<x_{1}<R$ (support of $\left.\theta_{R}\right)$ and $t \in(1 / 32,31 / 32)$. Hence, its contribution is bounded by (see (3.12) )

$$
\begin{aligned}
& c R^{4} \int_{1 / 32}^{31 / 32} \int_{\frac{1}{2}<x_{1}<R}|\widetilde{v}(x, t)|^{2} e^{2 \sigma(1+1 /(2 R))^{2}} d x d t \\
& \leq c R^{4} e^{2 \sigma(1+1 /(2 R))^{2}} \int_{1 / 32}^{31 / 32} \int_{\frac{1}{2}<x_{1}<R}|\widetilde{v}(x, t)|^{2} d x d t \\
& \leq c e^{2 \sigma(1+1 /(2 R))^{2}} \frac{A_{1}}{\delta^{n / 2}} R^{4} .
\end{aligned}
$$

Collecting this information and using that $R$ is large we get

$$
\frac{b}{2} c^{3} R^{2} e^{2 \sigma(1+1 / R)^{2}} \leq c \Xi(R) e^{2 \sigma(2-1 / R)^{2}}+c_{A_{1}, \delta} e^{2 \sigma(1+1 / 2 R)^{2}} R^{4} .
$$

Since $R$ is large the second term on the right hand side (3.39) can be hidden on the left to get that

$$
\frac{b}{4} c^{3} R^{2} e^{2 \sigma(1+1 / R)^{2}} \leq c \Xi(R) e^{2 \sigma(2-1 / R)^{2}} .
$$

Now, since $\sigma=c R^{2}$ one has that

$$
2 \sigma\left(1+\frac{1}{R}\right)^{2}-2 \sigma\left(2-\frac{1}{R}\right)^{2}=-6 c R^{2}+12 c R \geq-10 c R^{2},
$$

for $R$ large. Thus, from (3.40) it follows that

$$
\frac{b}{4} c^{3} R^{2} e^{-10 c R^{2}} \leq c \Xi(R) .
$$

But using (3.25)

$$
\begin{aligned}
\Xi(R) & =\int_{R-1<x_{1}<R} \int_{1 / 32}^{31 / 32}\left(|\widetilde{v}|^{2}+\left|\partial_{x_{1}} \widetilde{v}\right|^{2}\right)(x, t) d t d x \\
& =\int_{R-1<x_{1}<R} \int_{1 / 32}^{31 / 32} e^{2 \gamma \phi\left(x_{1}\right)} e^{-2 \gamma \phi\left(x_{1}\right)}\left(|\widetilde{v}|^{2}+\left|\partial_{x_{1}} \widetilde{v}\right|^{2}\right)(x, t) d t d x \\
& \leq c e^{-\gamma R} R^{3} \lambda e^{2 \lambda m / \delta^{1 / 2}} \leq c R^{3} e^{-\gamma R} e^{2 \lambda m / \delta^{1 / 2}},
\end{aligned}
$$

for $\lambda>>1$ and $x_{1}>R>>1$ one has that

$$
\frac{1}{2} x_{1} \leq \phi\left(x_{1}\right) \leq x_{1} .
$$

Thus, inserting (3.42) into (3.41) it follows that

$$
b \leq c e^{10 c R^{2}-\gamma R+3 \lambda m / \delta^{1 / 2}},
$$

where

$$
R=\frac{2 M \lambda^{1 / 2} m}{\left(\delta^{1 / 2} a_{1}\right)^{1 / 2} c}, \quad \gamma=\left(\lambda \delta^{1 / 2} a_{1}\right)^{1 / 2}, \quad \text { and } \quad \frac{2}{c_{n}} M \geq 1 / \delta^{1 / 2} .
$$

So we have, changing $c_{n}$ into $c$,

$$
\begin{aligned}
& 10 c R^{2}-\gamma R+3 \lambda m / \delta^{1 / 2} \\
& =\frac{40 c M^{2} m^{2} \lambda}{c^{2} \delta^{1 / 2} a_{1}}-\left(\lambda \delta^{1 / 2} a_{1}\right)^{1 / 2} \frac{2 M \lambda^{1 / 2} m}{\left(\delta^{1 / 2} a_{1}\right)^{1 / 2} c}+3 \frac{\lambda m}{\delta^{1 / 2}} \\
& =\lambda\left(\frac{40 c M^{2} m^{2}}{c^{2} \delta^{1 / 2} a_{1}}-\frac{2 M m}{c}+\frac{3 m}{\delta^{1 / 2}}\right) .
\end{aligned}
$$


We need the expression in parenthesis to be negative, i.e.

$$
\frac{40 c M^{2} m^{2}}{c^{2} \delta^{1 / 2} a_{1}}+\frac{3 m}{\delta^{1 / 2}}<\frac{2 M m}{c} .
$$

Divide by $M m$, we need

$$
\frac{40 c M m}{c^{2} \delta^{1 / 2} a_{1}}+\frac{3}{M \delta^{1 / 2}}<\frac{2}{c} .
$$

First, we choose $M$ so large such that $2 M / c \geq 1 / \delta^{1 / 2}$ and

So now we just need

$$
\frac{3}{M \delta^{1 / 2}} \leq \frac{1}{c} \text {. }
$$

$$
\frac{40 c M m}{c^{2} \delta^{1 / 2} a_{1}}<\frac{1}{c} .
$$

This can be done by taking $m>0$ small. Therefore, we have proved that $b=0$, which yields the desired result.

\section{REFERENCES}

[1] S. Ben Farah, and K. Mokni, Uncertainty principles and the $L^{p}-L^{q}$-version of Morgan's theorem on some groups, Russian J. Math. Physics 10 (2003) 245-260.

[2] H. Berestycki, and P.-L. Lions, Nonlinear scalar field equations, Arch. Rational Mech. Anal. 82 (1983) 313-375.

[3] A. Bonami, B. Demange, and P. Jaming, Hermite functions and uncertainty principles for the Fourier and the windowed Fourier transforms, Rev. Mat. Iberoamericana 19 (2006) 23-55.

[4] T. Carleman, Sur un probléme d'unicité pour les systémes d' equations aux derivées partielles á deux variables indépendantes, Ark. Math. 26B, (1939) 1-9.

[5] M. Cowling, and J. F. Price, Generalizations of Heisenberg's inequality, Harmonic Analysis (Cortona, 1982) Lecture Notes in Math.,992 (1983), 443-449, Springer, Berlin.

[6] L. Escauriaza, C. E. Kenig, G. Ponce, and L. Vega, On Uniqueness Properties of Solutions of Schrödinger Equations, Comm. PDE. 31, 12 (2006) 1811-1823.

[7] L. Escauriaza, C. E. Kenig, G. Ponce, and L. Vega, Hardy's Uncertainty Principle, Convexity and Schrödinger Evolutions, J. European Math. Soc. 10, 4 (2008) 883-907.

[8] L. Escauriaza, C. E. Kenig, G. Ponce, and L. Vega, The sharp Hardy Uncertainty Principle for Schrödinger evolutions, Duke Math. J. 155, (2010) 163-187.

[9] L. Escauriaza, C. E. Kenig, G. Ponce, and L. Vega, Uncertainty principle of Morgan type and Schrödinger evolution, J. London Math. Soc. 81, (2011) 187-207.

[10] L. Escauriaza, C. E. Kenig, G. Ponce, L. Vega, Uniqueness properties of solutions to Schrödinger equations, to appear in the Bulletin of the AMS.

[11] G. H. Hardy, A Theorem Concerning Fourier Transforms, J. London Math. Soc. s1-8 (1933) $227-231$.

[12] L. Hörmander, A uniqueness theorem of Beurling for Fourier transform pairs, Ark. Mat. 29, 2 (1991) 237-240.

[13] A. E. Ingham, A note on Fourier transforms J. London Math. Soc. s1-9 (1934) 29-32.

[14] A. D. Ionescu, and C. E. Kenig, L $L^{p}$-Carleman inequalities and uniqueness of solutions of nonlinear Schrödinger equations, Acta Math. 193, 2 (2004) 193-239.

[15] A. D. Ionescu, and C. E. Kenig, Uniqueness properties of solutions of Schrödinger equations, J. Funct. Anal. 232 (2006) 90-136.

[16] V. Izakov, Carleman type estimates in an anisotropic case and applications, J. Diff . Eqs. 105 (1993) 217-238.

[17] C. E. Kenig, G. Ponce, and L. Vega, On unique continuation for nonlinear Schrödinger equations, Comm. Pure Appl. Math. 60 (2002) 1247-1262.

[18] G. W. Morgan, A note on Fourier transforms, J. London Math. Soc. 9 (1934), 187-192.

[19] E. K. Naranayan, and S. K. Ray, Beurling's theorem in $\mathbb{R}^{n}$, preprint.

[20] R. Paley, and N. Wiener, Fourier transform in the complex domain, Amer. Math. Soc. Providence RI (1934). 
[21] A. Sitaram, M. Sundari, and S. Thangavelu, Uncertainty principles on certain Lie groups, Proc. Indian Acad. Sci. Math. Sci. 105 (1995), 135-151.

[22] W. A. Strauss, Existence of solitary waves in higher dimensions, Comm. Math. Phys. 55 (1977), 149-162.

[23] B. Y. Zhang, Unique continuation properties of the nonlinear Schrödinger equations, Proc. Roy. Soc. Edinburgh. 127 (1997) 191-205.

(C. E. Kenig) Department of Mathematics, University of Chicago, Chicago, Il. 60637, USA.

E-mail address: cek@math.uchicago.edu

(G. Ponce) Department of Mathematics, University of California, Santa Barbara, CA 93106, USA.

E-mail address: ponce@math.ucsb.edu

(L. Vega) UPV/EHU, Dpto. de Matemáticas, Apto. 644, 48080 Bilbao, Spain.

E-mail address: luis.vega@ehu.es 